\title{
Plasma brain-derived neurotrophic factor (BDNF) concentration and the BDNF Val66Met polymorphism in suicide: a prospective study in patients with depressive disorder
}

This article was published in the following Dove Press journal:

Pharmacogenomics and Personalized Medicine

Ming Ai'
Jun Wang'
Jianmei Chen'
Wo Wang'
Xiaoming Xu'
Yao Gan'
Xuemei Li'
Xinyuan Gou'
Jun Cao'
Zhen Lv'
Xiaorong Chen '
Hengguang Wang'
Qing Ma2,3
Li Kuang ',2
'Department of Psychiatry, First Affiliated
Hospital, Chongqing Medical University,
Chongqing, People's Republic of China;
${ }^{2}$ Mental Health Center, University-Town
Hospital of Chongqing Medical University,
Chongqing, People's Republic of China;
3Translational Pharmacology Research
Core, New York State Center of Excellence
in Bioinformatics and Life Sciences, School of
Pharmacy and Pharmaceutical Sciences,
University at Buffalo, Buffalo, NY, USA

Correspondence: Qing Ma Translational Pharmacology Research Core, New York State Center of Excellence in Bioinformatics and Life Sciences, School of Pharmacy and Pharmaceutical Sciences, University at Buffalo, Buffalo, NY 142।4, USA Email qingma@buffalo.edu

Li Kuang

Department of Psychiatry, First Affiliated Hospital, Chongqing Medical University, I You Yi Road, Yuzhong District, Chongqing 400016, People's Republic of China

Email kuangli0308@163.com
Purpose: The relationship was investigated between brain-derived neurotrophic factor (BDNF) concentrations, a $B D N F$ polymorphism $(196 \mathrm{G}>\mathrm{A})$, and the response to selective serotonin reuptake inhibitors (SSRIs) among Chinese patients with major depressive disorder (MDD).

Patients and methods: A total of 125 patients and 91 healthy controls were enrolled. The disease progression and treatment responses were evaluated using the Hamilton depression rating scale (HAMD24), the Hamilton anxiety scale, the Beck depression index, and BDNF concentrations at the baseline, 4, 8, and 12 weeks after treatment. Responders were defined as patients with at least a 50\% decrease in the HAMD24.

Results: The BDNF concentrations were significantly lower in MDD (947 \pm 297 vs 1187 $\pm 236 \mathrm{pg} / \mathrm{mL}, p=0.019$ ), in MDD with attempted suicide than those without (779 $\pm 231 \mathrm{vs} 993$ $\pm 298, p=0.024)$ at the baseline. The BDNF concentrations remarkably increased in response to SSRI treatment. Significant correlations were noted between the BDNF concentrations and suicide ideation or attempted suicide $(p<0.01)$, but not with HAMD24 or depression. BDNF $196 \mathrm{G}>$ A correlated with neither suicide ideation nor treatment responses.

Conclusion: BDNF concentrations were significantly lower in patients with attempted suicide/ideation. BDNF concentrations could serve as a response marker for antidepressant treatment in MDD.

Keywords: brain-derived neurotrophic factor, depression, polymorphism, attempted suicide, SSRI

\section{Introduction}

Suicide is the fifth leading cause of death in China. ${ }^{1}$ Approximately two-thirds of patients with depressive disorder have suicide ideation or attempt and up to $15 \%$ result in death. Suicide has increasingly become not only a significant issue of public health, but also a social and economic burden in China. ${ }^{2}$ The recent Global Burden of Disease Study 2010 has indicated that self-harm including suicide remains as one of the major disease burdens in China despite rapid health transition including remarkable declines in fertility and child mortality and increases in life expectancy during 1990-2010. ${ }^{3}$

Although the underlying mechanisms in the pathogenesis and treatment of major depressive disorder (MDD) for suicide prevention are not fully understood, there is considerable evidence suggesting that brain-derived neurotrophic factor (BDNF) and 
its receptor neurotrophic tyrosine kinase receptor type 2 might play an important role in mental disorders, particularly in depression, suicidal behavior, and antidepressant treatment-emergent suicidal ideation. ${ }^{4-6}$ BDNF is physiologically involved in neuronal survival, differentiation, and outgrowth during development and in adulthood. ${ }^{7,8}$ It serves as a mediator for the neuroplasticity of serotonergic and dopaminergic neurons, which have been implicated in suicidal behavior. ${ }^{9}$ Significantly lower BDNF concentrations have been reported in patients with depression, bipolar disorder, anxiety disorders, and schizophrenia that have a high suicide risk. ${ }^{10,11}$

The relationship between BDNF concentrations and antidepressant treatment has been investigated among patients with depression. Significant increases in BDNF concentrations were reported among depressed patients receiving antidepressant treatment, ${ }^{12,13}$ suggesting BDNF as a surrogate marker for treatment responses. Since BDNF is involved in neuronal plasticity, these results also parallel the neuroplastic improvement implied as an underlying mechanism of antidepressant treatment. Thus, BDNF might provide a protection against stress-induced neuronal impairment and modulation of BDNF levels would, therefore, be an important approach to be considered for therapeutic management of depression. ${ }^{10}$

The $B D N F$ gene might be associated with the response to antidepressant treatment, particularly selective serotonin reuptake inhibitors (SSRIs), since BDNF plays a significant role in the development of the serotonergic system. The $B D N F$ is located on the short arm of chromosome $11 \mathrm{p} 14$, and consists of 11 exons; encodes a precursor peptide that is cleaved to form the mature protein BDNF. ${ }^{14}$ One of the most investigated genetic variations within the $B D N F$ is a $196 \mathrm{G}>\mathrm{A}$ (rs6265) substitution, which results in a valine to methionine substitution at amino acid 66 (Val66Met) in the 5'proregion of the protein. ${ }^{15,16}$ Conflicting results have been reported regarding the association of this variation with antidepressant treatment. $^{17-19}$ The objective of this study was to determine the relationship between the Val66Met polymorphism in the BNDF gene and the response to antidepressant treatment among patients with suicide attempts or ideation.

\section{Patients and methods}

\section{Patients}

The study protocol was approved by the institutional review board of the Chongqing Medical University. Written informed consent was obtained from all participants. A total of 125 Han Chinese patients with depression were recruited from consecutive admissions to the Mental Health Center of Chongqing Medical University Hospital from September 2010 to November 2011. The inclusion criteria included: (1) age 18 or above, (2) meeting DSMIV and Chinese Classification and Diagnostic Criteria of Mental Disorder 3 (CCMD-3) criteria for depression, (3) not taking antidepressant medication within at least two weeks prior to the study, (4) having a 24-item Hamilton Rating Scale for Depression (HAMD-24) score greater than 20, and a Beck Self-Rating Depression Index (BDI) score greater than 5. Exclusion criteria included substance use disorders, pregnancy, menstruation, and physical and mental disorders that require immediate treatment. Healthy volunteers in the Control group $(n=91)$ were recruited from Chongqing Medical University Hospital and the medical school with matched age and gender, HAMD-24 below 8 and no substance abuse history or mental disorders. All experiments on human subjects were conducted in accordance with the Declaration of Helsinki.

\section{Clinical assessments}

Demographics, family depression histories, and previous antidepressant treatment courses, including the dose and treatment duration, and suicide attempts were obtained from medical records. The patients were treated with daily SSRI, eg, fluoxetine or paroxetine, or SSRI with low dose atypical antipsychotics, eg, olanzapine daily, for 12 weeks. The clinical assessments were conducted by two experienced psychiatrists using the HAMD-24 and BDI before and at 4,8 , and 12 weeks after the antidepressant treatment. The higher the HAMD-24 or the BDI scores were, the more severe depressive symptoms were reported from patients. The medication adherence was assessed by self-report and pill counting by pharmacists. A regular weekly phone reminder was used to assure adherence. Treatment responders were defined as patients with an at least $50 \%$ decrease in the HAMD-24 at week 12, whereas the rest of patients were considered to be non-responders. ${ }^{20,21}$

\section{Plasma BDNF measurement}

Samples of $4 \mathrm{~mL}$ of blood were collected at the baseline and during follow-up visits at 4, 8, and 12 weeks. The blood samples were then centrifuged to obtain plasma for BDNF measurement and blood cells for genomic DNA extraction. Plasma concentrations of BDNF were measured using the Human BDNF Immunoassay Quantikine ${ }^{\circledR}$ ELISA Kit (R\&D Systems GmbH, Wiesbaden-Nordenstadt, Germany). 
ELISA assays were performed according to the manufacturer's instructions. Briefly, the plates were pre-coated with the mouse monoclonal antibody against BDNF. Fifty microliters of standards and samples was added in duplicate, and incubated for 2 hrs. After an addition of BDNF Conjugate and a $1 \mathrm{hr}$ incubation period, wells were washed extensively with washing buffer. Two hundred microliters of the Substrate Solution was added followed by 30 mins incubation. Reaction was stopped by adding $50 \mu \mathrm{L}$ of Stop Solution and absorbance was determined within $30 \mathrm{mins}$ at $450 \mathrm{~nm}$ using an ELISA plate reader. The minimum detectable dose of BDNF as reported by the manufacturer was $20 \mathrm{pg} / \mathrm{mL}$.

\section{Genotyping}

Genomic DNA was isolated from the blood cells using standard procedures. BDNF 196G $>$ A polymorphism (rs6265) was genotyped by the PCR-RFLP method. ${ }^{22}$ PCR amplification was performed with primers (forward 5'-AAA CAT CCG AGG ACA AGG TG-3' and reverse 5'-AGA AGA GGA GGC TCC AAA GG-3', GenScript, Nanjing, China), 2 X Es Taq Master Mix (CoWin Bioscience Co., Ltd., Beijing, China), and 50-100 ng genomic DNA in a final volume of $50 \mu \mathrm{l}$. After activation of DNA polymerase for 2 mins at $94^{\circ} \mathrm{C}$, the reaction mixture was subjected to 35 cycles of $94^{\circ} \mathrm{C}$ for $30 \mathrm{sec}, 60^{\circ} \mathrm{C}$ for $30 \mathrm{sec}, 72^{\circ} \mathrm{C}$ for $30 \mathrm{sec}$, followed by a final extension at $72^{\circ} \mathrm{C}$ for 2 mins. The amplification product was digested with Pml I (Fermentas China, Shenzhen, China) at $37^{\circ} \mathrm{C}$ for 5 mins, according to the manufacturer's instructions. The resulting DNA fragments were separated on a $3 \%$ agarose gel. While the GG and AA genotype showed a band at 125 and 249 bp, respectively, the AG genotype showed two bands at 125 and 249 bp.

\section{Statistics}

All statistical analyses were conducted using SPSS 17.0 (SPSS, Inc., Chicago, IL). The clinical characteristics between patients and control groups were compared by the Student's $t$-test or the nonparametric Mann-Whitney
$U$-test. The differences in BDNF concentrations among different patient and control groups were evaluated using one-way ANOVA. Correction for multiple testing was performed by calculation of adjusted $p$-values. The Hardy-Weinberg equilibrium test and incidence analyses were performed using a modified Chi-square test. The Spearman's correlation coefficient was used to assess the relationship between the genotype or allele type and different phenotypes. The statistical significance was set at $p$ values less than 0.05 .

\section{Results}

\section{Characteristics of the subjects}

The demographic and clinical characteristics of the depressed patients and the control group are summarized in Table 1. There were no significant differences in age, sex, education, or family history between two groups. The average plasma BDNF concentration of depressed patients was significantly lower than that of normal controls (947 vs $1187 \mathrm{pg} / \mathrm{mL}, p=0.019$ ). No significant difference in BDNF concentrations was noted between the male and female patients. A detailed comparison of baseline BDNF concentrations is summarized in Table 2. Significantly lower BDNF concentrations were observed in patients with previous suicide attempts $(p=0.024)$. Low BDNF levels were also noted in patients with suicide ideation, suicide family history, and recurrent depression without statistical significance, except late-onset depression $(p=0.046)$.

Clinical assessment including HAMD and BDI is summarized in Table 3 . There were no significant differences in HAMD or BDI between genders, groups with/ without family history, early- and late-onset depression, and newly diagnosed and recurrent depression. A trend of higher HAMD was observed in patients with previous suicide attempts without reaching statistical significance $(p=0.076)$. Significantly higher HAMD and BDI were noted in patients with suicide ideation $(p<0.01)$.

Table I Demographic and clinical characteristics of depressed patients and normal controls

\begin{tabular}{|l|l|l|l|}
\hline & Depressed patients $(\mathbf{n = 1 2 5})$ & Normal controls $(\mathbf{n = 9} \mathbf{I})$ & $\boldsymbol{P}$ \\
\hline Age - year, mean \pm SD & $42.9 \pm 16.1$ & $41.2 \pm 15.5$ & 0.432 \\
Sex (male/female) & $49 / 76$ & $36 / 55$ & 0.826 \\
Education $^{\text {a }}$ & $9 / 42 / 30 / 44$ & $7 / 29 / 22 / 33$ & 0.988 \\
Family history (no/yes) & $89 / 36$ & $73 / 18$ & 0.550 \\
Baseline BDNF level - Pg/mL, mean \pm SD & $947 \pm 297$ & $1187 \pm 236$ & 0.019 \\
\hline
\end{tabular}

Note: ${ }^{a}$ Education: primary school/middle school/high school/college. 
Table 2 Comparison of BDNF concentrations at the baseline $(\mathrm{pg} / \mathrm{mL}$, mean $\pm \mathrm{SD})$

\begin{tabular}{|l|l|l|l|l|}
\hline & & N & BDNF & $\boldsymbol{p}$ \\
\hline Family history & Yes & 36 & $925 \pm 294$ & 0.553 \\
& No & 89 & $1,002 \pm 306$ & \\
\hline Suicide attempts & Yes & 27 & $\begin{array}{l}779 \pm 231 \\
993 \pm 298\end{array}$ & 0.024 \\
\hline No & 98 & 84 & $895 \pm 291$ & 0.925 \\
\hline Suicide ideation & Yes & $4,053 \pm 284$ & \\
\hline Early onset <30 years & Yes & 39 & $969 \pm 333$ & 0.046 \\
& No & 86 & $932 \pm 275$ & \\
\hline Newly diagnosed & Yes & 68 & $989 \pm 302$ & 0.310 \\
& No & 57 & $896 \pm 285$ & \\
\hline
\end{tabular}

Abbreviation: BDNF, brain-derived neurotrophic factor.

\section{Responses to antidepressant treatment}

Therapeutic responses to antidepressant treatment, primarily including paroxetine and fluoxetine, were measured by the relative intra-individual changes of $\mathrm{HAMD} / \mathrm{BDI}$ and BDNF concentrations over the 12-week study period. Sixty-one of the 125 depressed patients (49\%) had complete BDNF concentration profiles that were included in the final analysis. HAMD/BDI depression ratings significantly improved with antidepressant treatment after 4-week treatment, and a continuous improvement was noted during the 12-week study period (Table 4). Significantly lower BDNF concentrations were observed among patients with depressive disorders in comparison to those from healthy controls $(784 \pm 165$ vs $1187 \pm 236 \mathrm{pg} / \mathrm{mL}, p<0.01)$ at the baseline, which increased to $1040 \pm 256,1103 \pm 301$, and $1257 \pm 254$ $\mathrm{pg} / \mathrm{mL}$ by the end of $4-, 8$ - and 12-week antidepressant treatment, respectively.

Correlation analyses revealed a significant association between the baseline BDNF concentrations with BDI ratings ( $r=-0.196, p=0.029)$, suicide ideation $(r=-0.250, p=0.005)$, and previous suicide attempts $(r=-0.298, p<0.0001)$, but not with gender, age, family history, early onset, recurrence, or HAMD ratings. However, this association became insignificant 4 weeks after antidepressant treatment and remained insignificant by the end of the 12-week study period.

\section{BDNF Val66met polymorphism, depression, and suicide attempts}

The genotypes and allele distributions for the $B D N F$ Val66Met polymorphism for the depressed patients and controls are presented in Table 5. The genotype distributions for these two groups were in the Hardy-Weinberg equilibrium $\left(\chi^{2}=0.393\right.$, $p=0.531 ; \chi^{2}=2.528, p=0.112$, for depressed patients and controls, respectively). Neither genotype $(p=0.194)$ nor allelic frequencies $(p=0.121)$ were statistically different between the two groups. No relationship was observed between BDNF genotypes and quantitative measures of depression, including early onset ( $<30$ years) and recurrence, gender, family history, or previous suicide attempts (Table 6).

Table 3 Comparison of HAMD and BDI scales at the baseline (mean \pm SD)

\begin{tabular}{|l|l|l|l|l|l|}
\hline & & HAMD & $\boldsymbol{P}$ & BDI & P \\
\hline Gender & Male & $25.2 \pm 4.7$ & $25.6 \pm 5.0$ & $\begin{array}{l}17.2 \pm 6.1 \\
16.6 \pm 7.0\end{array}$ & 0.527 \\
& Female & $26.9 \pm 3.5$ & $24.1 \pm 5.2$ & $17.5 \pm 6.7$ \\
\hline Family history & Yes & 0.382 & $14.5 \pm 6.3$ \\
\hline No & Yes & $29.3 \pm 5.0$ & $22.4 \pm 6.4$ & $15.3 \pm 5.9$ \\
\hline Suicide attempts & No & $24.4 \pm 4.2$ & 0.076 & $18.4 \pm 7.0$ \\
& Yes & $26.2 \pm 5.3$ & 0.001 & $13.5 \pm 4.5$ \\
\hline Early onset <30 years & No & $23.9 \pm 3.3$ & $26.1 \pm 5.0$ & $19.1 \pm 6.4$ & $15.8 \pm 6.6$ \\
\hline Newly diagnosed & Yes & $25.2 \pm 4.8$ & 0.327 & $17.3 \pm 6.8$ \\
& No & $25.2 \pm 5.0$ & 0.689 & $16.3 \pm 6.6$ \\
\hline
\end{tabular}

Abbreviations: HAMD, Hamilton Rating Scale for Depression; BDI, Beck Self-Rating Depression Index. 
Table 4 Responses to antidepressant treatment (mean \pm SD)

\begin{tabular}{|l|l|l|l|l|}
\hline \multirow{2}{*}{} & \multirow{2}{*}{ Baseline } & \multicolumn{4}{|l|}{ Post-antidepressant treatment (weeks) } \\
\cline { 3 - 5 } & & $\mathbf{4}$ & $\mathbf{8}$ & $\mathbf{1 2}$ \\
\hline HAMD & $24.9 \pm 4.3$ & $16.8 \pm 5.0^{*}$ & $11.3 \pm 3.5^{*}$ & $7.3 \pm 2.3^{*}$ \\
BDI & $16.8 \pm 6.2$ & $12.9 \pm 6.9^{*}$ & $8.4 \pm 3.8^{*}$ & $5.3 \pm 3.0^{*}$ \\
BDNF $(\mathrm{pg} / \mathrm{mL})$ & $784 \pm 165$ & $1040 \pm 256^{*}$ & $1103 \pm 301^{*}$ & $1257 \pm 254^{*}$ \\
\hline
\end{tabular}

Note: $* P<0.01$, compared to the baseline ratings.

Abbreviations: HAMD, Hamilton Rating Scale for Depression; BDI, Beck Self-Rating Depression Index; BDNF, brain-derived neurotrophic factor.

Table 5 BDNF genotype distributions and allele frequency in 125 depressed patients and 9 I control subjects

\begin{tabular}{|l|l|l|l|l|l|l|l|l|}
\hline & \multirow{2}{*}{} & & \multicolumn{2}{|l|}{ Genotypes - n (\%) } & \multicolumn{3}{l|}{ Allele frequencies (\%) } \\
\cline { 3 - 9 } & $\mathbf{n}$ & Val/Val & Val/Met & Met/Met & $\boldsymbol{P}$ & Val & Met & $\boldsymbol{P}$ \\
\hline Patients & 125 & $30(24.0)$ & $66(52.8)$ & $29(23.2)$ & 0.194 & 50.4 & 49.6 & 0.121 \\
Controls & 91 & $13(28.6)$ & $52(57.1)$ & $26(14.3)$ & & 42.9 & 57.1 & \\
\hline
\end{tabular}

Table 6 Demographic data and clinical characteristics of the BDNF genotype groups within the depressed patients

\begin{tabular}{|c|c|c|c|c|c|}
\hline & \multirow[t]{2}{*}{$\mathbf{n}$} & \multicolumn{3}{|c|}{ Genotypes } & \multirow[t]{2}{*}{$p$} \\
\hline & & Val/Val & Val/Met & Met/Met & \\
\hline Gender (M/F) & $49 / 76$ & $11 / 19$ & $28 / 38$ & $10 / 19$ & 0.726 \\
\hline Family history $(\mathrm{Y} / \mathrm{N})$ & $36 / 89$ & $8 / 22$ & $20 / 46$ & $8 / 21$ & 0.923 \\
\hline Early onset $(\mathrm{Y} / \mathrm{N})$ & $39 / 86$ & $9 / 21$ & $21 / 45$ & $9 / 20$ & 0.941 \\
\hline Recurrence $(\mathrm{Y} / \mathrm{N})$ & $57 / 68$ & $16 / 14$ & $29 / 37$ & $12 / 17$ & 0.605 \\
\hline Previous suicide attempts $(\mathrm{Y} / \mathrm{N})$ & $27 / 98$ & $7 / 23$ & $15 / 55$ & $5 / 24$ & 0.807 \\
\hline
\end{tabular}

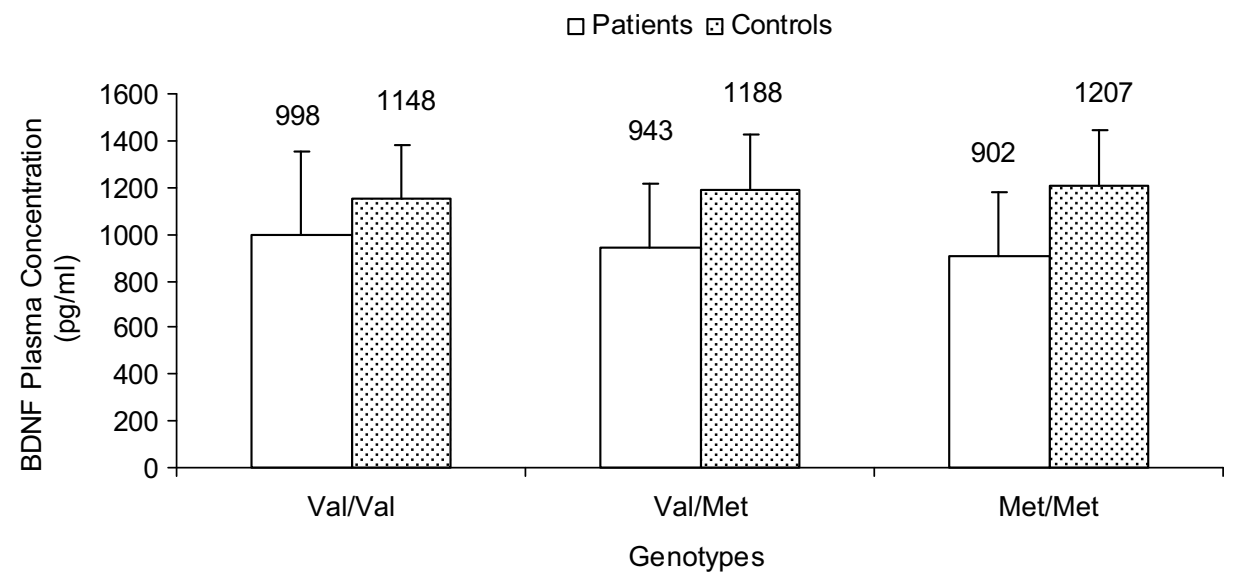

Figure I BDNF concentration in plasma by BDNF Val66Met genotypes. Analyses of variance indicates no significant differences between groups.

Interaction between BDNF Val66met polymorphism, BDNF plasma concentrations and treatment responses

BDNF plasma concentrations at baseline by genotypes are presented in Figure 1. There were no significant differences in BDNF concentrations among the three genotype groups ( $p=0.462, p=0.764$ for patients and controls, respectively). BDNF concentrations from 61 patients in response to antidepressant treatment over the 12 -week study period are summarized in Table 7 by 
Table 7 Plasma BDNF concentrations in response to antidepressant treatment by the $B D N F$ genotype groups $(\mathrm{pg} / \mathrm{mL}$, mean $\pm S D)$

\begin{tabular}{|l|l|l|l|l|}
\hline \multirow{2}{*}{ Time (week) } & \multicolumn{2}{|l|}{ Genotypes } & \multirow{2}{*}{$\boldsymbol{P}$} \\
\cline { 2 - 4 } & Val/Val & Val/Met & Met/Met & \\
\hline 0 (baseline) & $762 \pm 162$ & $802 \pm 156$ & $772 \pm 183$ & 0.666 \\
4 & $858 \pm 210$ & $1002 \pm 196$ & $941 \pm 226$ & 0.190 \\
8 & $1020 \pm 259$ & $1161 \pm 199$ & $1088 \pm 209$ & 0.178 \\
12 & $1224 \pm 127$ & $1292 \pm 229$ & $1235 \pm 170$ & 0.514 \\
\hline
\end{tabular}

Abbreviation: BDNF, brain-derived neurotrophic factor.

genotypes. BDNF concentrations significantly increased from baseline $(\sim 770 \mathrm{pg} / \mathrm{mL})$ to $\sim 1235 \mathrm{pg} / \mathrm{mL}$ at Week 12 $(p<0.001)$. This increase was statistically significant within each genotype group. Genotype-associated increases, between $\mathrm{Val} / \mathrm{Val}$, Val/Met, and Met/Met, in plasma BDNF concentrations did not significantly differ (Table 7).

The correlation between BDNF genotypes, antidepressant response at week 12 , and treatment options was examined in the patient subgroups: 43 patients were responders, and 18 patients were non-responders; 24 were on SSRI, and 37 were on SSRI plus a low dose of atypical antipsychotic drugs (SSRI+AA). The genotype and allele, distributions are summarized in Table 8. No significant differences were noted between responders and non-responders, or between SSRI and SSRI+AA groups.

\section{Discussion}

MDDs have a large social and economic impact that is associated with suicide, increased morbidity and mortality. There are several lines of evidence suggesting a potential role of BDNF in the pathogenesis and treatment of a variety of mental illnesses, such as MDD, ${ }^{23,24}$ schizophrenia, ${ }^{25}$, and bipolar disorder. ${ }^{26,27}$ Our previous studies in Chinese patients with anxiety/depression and schizophrenia demonstrated significantly lower BDNF concentrations among these patients than those in the control. ${ }^{28,29}$ The overall objective of these studies, including the present one, was to evaluate BDNF as a predictive biomarker for diagnosis and treatment of depression and schizophrenia with a particular reference to suicide. The main findings of this study were observed significant associations between plasma BDNF concentrations, early onset depression, suicide attempts and ideation, and responses to antidepressant treatment. There was an observed lack of associations between the Val66Met (rs6265) polymorphism and the antidepressant responses, or between BDNF concentrations and suicide at 12 weeks after antidepressant treatment.

\section{Plasma BDNF concentrations and depression}

Significantly lower plasma BDNF concentrations were observed among depressed patients in comparison to those of the control, which was consistent with the results from previous studies. ${ }^{11,30-32}$ In the subgroup with a history of suicide attempts, the plasma BDNF levels were significantly lower than those without suicidal behavior; depression and suicidal ideation were associated with plasma BDNF levels. These findings were consistent with previous observations indicating low BDNF concentrations among Korean patients with severe depression and suicidal ideation. ${ }^{33,34}$ To our knowledge, the present study represented the first report on the relationship between BDNF levels and suicide among Chinese patients. Our results also suggested that commonly used clinical assessments, including HAMD and BDI, might not be sufficient to identify suicidal behavior because no association was observed between the average scores of HAMD/BDI and suicide, which was consistent to the previous observations indicating lack of clinical utility of these scales in predicting suicide. ${ }^{35}$ A combination of clinical assessments of

Table 8 Correlation of genotypes and alleles of the BDNF Val66Met polymorphism, antidepressant responses at week 12 and treatment options

\begin{tabular}{|c|c|c|c|c|c|c|c|c|}
\hline & \multirow[b]{2}{*}{$\mathbf{n}$} & \multicolumn{4}{|c|}{ Genotypes - n (\%) } & \multicolumn{3}{|c|}{ Allele frequencies (\%) } \\
\hline & & Val/Val & Val/Met & Met/Met & $p$ & Val & Met & $p$ \\
\hline Responders & 43 & $8(18.6)$ & $18(41.9)$ & $17(39.5)$ & 0.426 & $34(39.5)$ & $52(60.5)$ & 0.286 \\
\hline Non-responders & 18 & $4(22.2)$ & $10(55.6)$ & $4(22.2)$ & & $18(50.0)$ & $18(50.0)$ & \\
\hline SSRI & 24 & $3(12.5)$ & $10(41.7)$ & II (45.8) & 0.261 & $16(33.3)$ & $32(66.7)$ & 0.095 \\
\hline SSRI+AA & 37 & $9(24.3)$ & I 8 (48.7) & $10(27.0)$ & & $36(48.6)$ & $38(5 \mid .4)$ & \\
\hline
\end{tabular}

Abbreviations: BDNF, brain-derived neurotrophic factor; SSRI, selective serotonin reuptake inhibitor. 
depression and the plasma BDNF concentrations might provide a better approach for prediction and prevention of suicide in patients with depressive disorders.

The lack of significant association between plasma BDNF concentrations with gender, age, family history, HAMD, disease duration, recurrence or early onset observed in the present study was consistent to the previous observations among Chinese patients. ${ }^{36,37}$ The BDNF concentrations were significantly correlated with self-reported depressive symptoms as indicated by BDI, which was consistent to the previous results reported in the elderly patients with depression. ${ }^{38}$ In the present study, low BDNF concentrations were noted among patients with recurrence in comparison to that with the single episode, but the difference was not significant. This was inconsistent with previous results showing low BDNF levels were related to both recurrence and severity of depression, ${ }^{39,40}$ which might largely due to the relatively small sample size and different study populations. In fact, a large inter-patient variation of BDNF levels has been recently reported, ${ }^{41}$ suggesting a potential barrier for applying BDNF levels as a surrogate marker.

A significant and sequential increase in plasma BDNF concentrations from the baseline was noted in the present study at 12 weeks after antidepressant treatment initiation. This finding was consistent with the previous reports indicating increased BDNF levels from the baseline compared to the control after one-month antidepressant treatment, as well as remarkable improvement in depression at 48 weeks of treatment. ${ }^{42}$ However, no correlation between BDNF concentrations at week 4 and 8 , antidepressant effects or prognosis was observed in our study, suggesting extended follow-up for a longer time period might help define the relationship of biomarkers, eg, BNDF concentrations, and antidepressant responses. Unlike the previous finding by Reiji et al, that demonstrated a significant increase of BDNF concentrations after addition of atypical antipsychotics at low doses into the SSRIs or mood stabilizers-based regimens among patients with refractory depression, ${ }^{20}$ minimal impact of antipsychotics was observed in the present study likely due to the differences in disease severity and treatment regimens. It is largely unclear if the refractory depression and general depressive disorders share the same pathogenesis, or the similar mechanism in response to antidepressant treatment. A slow elevation of BDNF concentrations was observed between 4 and 8 weeks after treatment initiation followed a rapid increase phase till 12 weeks, suggesting BDNF as a delayed response marker, which is consistent to a previous report indicating a slow increase phase at week $6 .{ }^{40}$ Unlike HAMD or BDI where no differences were noted between treatment and control groups by the end of week 12, the plasma BDNF concentrations in the treatment group were significantly higher than those in the control, reinforcing its potential as a treatment response marker. However, it remained unclear if treatment modifications, such as dosage adjustment, or termination should be based on changes of BDNF due to the short study period of 12 weeks that warrants a long-term multiple center clinical trial.

\section{BDNF 196G >A polymorphism and treatment responses}

No significant difference was noted between the treatment and control groups in frequencies of $B D N F 196 \mathrm{G}>\mathrm{A}$ polymorphism in the present study, confirming previous results among subjects in China and Taiwan. ${ }^{23,36,37}$ Racial differences have been demonstrated that Asians have a low $G$ allele frequency between $50 \%$ and $59 \%$, in comparison to that of Caucasians, between $77 \%$ and $83 \%$. However, since depression is a disease involving multiple genes and environmental factors, it is likely such a difference might not be directly translated into variations in treatment response. A recent study with a similar design ${ }^{43}$ has identified gender instead of $B D N F$ genotypes as the contributing factor, suggesting an important role of phenotypic heterogeneity. The possible phenotypic heterogeneity was also suggested by the exclusionary finding that no association was identified between $B D N F 196 \mathrm{G}>\mathrm{A}$, family history, early- or late-onset depression, attempted suicide, HAMD or BDI scores.

In addition, $B D N F 196 \mathrm{G}>\mathrm{A}$ appeared to have a limited impact on responses to an antidepressant alone or antidepressant with a small dose of antipsychotics. The results from the pharmacogenetics studies on $B D N F \quad 196 \mathrm{G}>\mathrm{A}$ were rather inconclusive. Although significantly better responses to paroxetine were reported among Chinese with MDD and BDNF 196A allele, no association was identified between this polymorphism and serum BDNF concentrations or response to SSRIs in depressed Japanese patients. $^{21,44}$ A comparative study demonstrated a significant association of $B D N F$ Val66Met genotype with responses to venlafaxine, but not to fluoxetine, suggesting differential impact of $B D N F$ polymorphisms on responses to antidepressants. ${ }^{45}$ The future study would be expanded to investigate the relationship between $B D N F$ and other 
antidepressants with different pharmacological mechanisms.

\section{Limitations}

There were several limitations to the present study. The sample size was relatively small and stratification into the subgroups with decreased observed concentration assessment further hampered the analysis. However, considering the difficulties in enrollment and follow-up of patients with MDD, the sample size was comparable to most recently published reports. ${ }^{21,23,44}$ The patient population was limited among Chinese primarily from Southwest China that a comprehensive inclusion of Chinese from different regions with different racial, educational and disease background would be optimal for generalization of the findings from the study. A longer follow-up, 1-2 years, with multiple BDNF concentration measurements would be an ideal methodologic approach to evaluate BDNF as a biomarker for depression treatment and prognosis. Lastly, early identification of suicide ideation and suicide prevention might benefit more from a clear understanding of the relationship between BDNF and attempted suicide in patients with MDD, especially those with suicidal ideation during a long-term follow-up study.

\section{Conclusion}

The utilization of BDNF concentrations as a biomarker for suicide and suicide ideation was supported by the observation of significantly lower BDNF concentrations in patients with attempted suicide. The prognostic quality of BDNF concentrations in MDD should be explored prospectively in larger clinical evaluations.

\section{Abbreviation list}

BDNF, brain-derived neurotrophic factor; Val, valine; Met, methionine; SSRI, selective serotonin reuptake inhibitor; MDD, major depressive disorders.

\section{Acknowledgments}

This work was supported by the National Natural Science Foundation Project of China (81671360), the Special Key Projects of Scientific and Technological Innovation for People's Livelihood in Chongqing (CSTC2017SHMSZDYF0128, CSTC2018JCYJAX0164), the Medical Research Project of Chongqing Medical Planning Commission (2018QNXM014, 2017MSXM016) and the Scientific Research and Cultivation Project of the First
Affiliated Hospital of Chongqing Medical University (PYJJ2018-20). The authors appreciated the invaluable support of Chen-Zhong Xu and $\mathrm{Ke} \mathrm{Li}$ with the data collection and Xiao-Ni Zhong, Wen-Feng Song and Bo Mi for their statistical support.

\section{Disclosure}

Qing Ma was supported in part by the National Institute of Health (NIH) K08MH098794. The authors report no other conflicts of interest in this work.

\section{References}

1. Wang Z, Wang J, Bao J, Gao X, Yu C, Xiang H. Temporal trends of suicide mortality in mainland China: results from the age-periodCohort framework. Int J Environ Res Public Health. 2016;13(8). doi:10.3390/ijerph13121252

2. Phillips MR, Yang G, Li S, Li Y. Suicide and the unique prevalence pattern of schizophrenia in mainland China: a retrospective observational study. Lancet. 2004;364(9439):1062-1068. doi:10.1016/ S0140-6736(04)17061-X

3. Yang G, Wang Y, Zeng Y, et al. Rapid health transition in China, 1990-2010: findings from the Global Burden of Disease Study 2010. Lancet. 2013;381(9882):1987-2015. doi:10.1016/S0140-6736(13) 61097-1

4. Duffy A, Grof P, Robertson C, Alda M. The implications of genetics studies of major mood disorders for clinical practice. J Clin Psychiatry. 2000;61(9):630-637.

5. Perroud N. Suicidal ideation during antidepressant treatment: do genetic predictors exist? CNS Drugs. 2011;25(6):459-471. doi:10.2165/11589420-000000000-00000

6. Perroud N, Aitchison KJ, Uher R, et al. Genetic predictors of increase in suicidal ideation during antidepressant treatment in the GENDEP project. Neuropsychopharmacology. 2009;34(12):2517-2528. doi:10.1038/ npp. 2009.81

7. Huang EJ, Reichardt LF. Neurotrophins: roles in neuronal development and function. Annu Rev Neurosci. 2001;24:677-736. doi:10.1146/annurev.neuro.24.1.677

8. Schinder AF, Poo M. The neurotrophin hypothesis for synaptic plasticity. Trends Neurosci. 2000;23(12):639-645.

9. Lu B, Nagappan G, Lu Y. BDNF and synaptic plasticity, cognitive function, and dysfunction. Handb Exp Pharmacol. 2014;220:223250. doi:10.1007/978-3-642-45106-5_9

10. Autry AE, Monteggia LM. Brain-derived neurotrophic factor and neuropsychiatric disorders. Pharmacol Rev. 2012;64(2):238-258. doi:10.1124/pr.111.005108

11. Lopresti AL, Maker GL, Hood SD, Drummond PD. A review of peripheral biomarkers in major depression: the potential of inflammatory and oxidative stress biomarkers. Prog Neuropsychopharmacol Biol Psychiatry. 2014;48:102-111. doi:10.1016/j.pnpbp.2013.09.017

12. Aydemir O, Deveci A, Taneli F. The effect of chronic antidepressant treatment on serum brain-derived neurotrophic factor levels in depressed patients: a preliminary study. Prog Neuropsychopharmacol Biol Psychiatry. 2005;29(2):261-265. doi:10.1016/j.pnpbp.2004.11.009

13. Gonul AS, Akdeniz F, Taneli F, Donat O, Eker C, Vahip S. Effect of treatment on serum brain-derived neurotrophic factor levels in depressed patients. Eur Arch Psychiatry Clin Neurosci. 2005;255 (6):381-386. doi:10.1007/s00406-005-0578-6

14. Mowla SJ, Farhadi HF, Pareek S, et al. Biosynthesis and post-translational processing of the precursor to brain-derived neurotrophic factor. $J$ Biol Chem. 2001;276(16):12660-12666. doi:10.1074/jbc.M008104200 
15. Egan MF, Weinberger DR, Lu B. Schizophrenia, III: brain-derived neurotropic factor and genetic risk. Am J Psychiatry. 2003;160 (7):1242. doi:10.1176/appi.ajp.160.7.1242

16. Hariri AR, Goldberg TE, Mattay VS, et al. Brain-derived neurotrophic factor val66met polymorphism affects human memory-related hippocampal activity and predicts memory performance. $J$ Neurosci. 2003;23(17):6690-6694.

17. Domschke K, Lawford B, Laje G, et al. Brain-derived neurotrophic factor (BDNF) gene: no major impact on antidepressant treatment response. Int $J$ Neuropsychopharmacol. 2010;13(1):93-101. doi:10.1017/S1461145709000030

18. Niitsu T, Fabbri C, Bentini F, Serretti A. Pharmacogenetics in major depression: a comprehensive meta-analysis. Prog Neuropsychopharmacol Biol Psychiatry. 2013;45:183-194. doi:10.1016/j.pnpbp. 2013.05.011

19. Su N, Zhang L, Fei F, et al. The brain-derived neurotrophic factor is associated with alcohol dependence-related depression and antidepressant response. Brain Res. 2011;1415:119-126. doi:10.1016/j. brainres.2011.08.005

20. Yoshimura R, Ikenouchi-Sugita A, Hori H, et al. Adding a low dose atypical antipsychotic drug to an antidepressant induced a rapid increase of plasma brain-derived neurotrophic factor levels in patients with treatment-resistant depression. Prog Neuropsychopharmacol Biol Psychiatry. 2010;34(2):308-312. doi:10.1016/j.pnpbp.2009.12.003

21. Yoshimura R, Kishi T, Suzuki A, et al. The brain-derived neurotrophic factor (BDNF) polymorphism Val66Met is associated with neither serum BDNF level nor response to selective serotonin reuptake inhibitors in depressed Japanese patients. Prog Neuropsychopharmacol Biol Psychiatry. 2011;35(4):1022-1025. doi:10.1016/j. pnpbp.2011.02.009

22. Matsushita S, Kimura M, Miyakawa T, et al. Association study of brain-derived neurotrophic factor gene polymorphism and alcoholism. Alcohol Clin Exp Res. 2004;28(11):1609-1612.

23. Tsai SJ, Cheng CY, Yu YW, Chen TJ, Hong CJ. Association study of a brain-derived neurotrophic-factor genetic polymorphism and major depressive disorders, symptomatology, and antidepressant response. Am J Med Genet Part B, Neuropsychiatr Genet. 2003;123B(1):1922. doi:10.1002/ajmg.b.20026

24. Tsai SJ, Hong CJ, Liou YJ. Brain-derived neurotrophic factor and antidepressant action: another piece of evidence from pharmacogenetics. Pharmacogenomics. 2008;9(9):1353-1358. doi:10.2217/ 14622416.9.9.1353

25. Grillo RW, Ottoni GL, Leke R, Souza DO, Portela LV, Lara DR. Reduced serum BDNF levels in schizophrenic patients on clozapine or typical antipsychotics. J Psychiatr Res. 2007;41(1-2):31-35. doi:10.1016/j.jpsychires.2006.01.005

26. Cunha AB, Frey BN, Andreazza AC, et al. Serum brain-derived neurotrophic factor is decreased in bipolar disorder during depressive and manic episodes. Neurosci Lett. 2006;398(3):215-219. doi:10.1016/j.neulet.2005.12.085

27. Pandey GN, Rizavi HS, Dwivedi Y, Pavuluri MN. Brain-derived neurotrophic factor gene expression in pediatric bipolar disorder: effects of treatment and clinical response. J Am Acad Child Adolesc Psychiatry. 2008;47(9):1077-1085. doi:10.1097/CHI.0b013e31817eecd9

28. Zeng Y, Ai M, Chen J, Kuang L. Correlation between trait anxiety and serum brain-derived neurotrophic factor level in patients with combined anxiety and depression. J Third Mil Med Univ. 2011;33 (18):1967-1969.

29. Zeng Y, Kuang L, Gan Y, et al. Expression and significance of plasma BDNF in patients with schizophrenia. J Chongqing Med Univ. 2011;4:476-478.
30. Kobayakawa M, Inagaki M, Fujimori M, et al. Serum brain-derived neurotrophic factor and antidepressant-naive major depression after lung cancer diagnosis. Jpn J Clin Oncol. 2011;41(10):1233-1237. doi:10.1093/jjco/hyr119

31. Rojas PS, Fritsch R, Rojas RA, Jara P, Fiedler JL. Serum brainderived neurotrophic factor and glucocorticoid receptor levels in lymphocytes as markers of antidepressant response in major depressive patients: a pilot study. Psychiatry Res. 2011;189(2):239-245. doi:10.1016/j.psychres.2011.04.032

32. Wolkowitz OM, Wolf J, Shelly W, et al. Serum BDNF levels before treatment predict SSRI response in depression. Prog Neuropsychopharmacol Biol Psychiatry. 2011;35(7):1623-1630. doi:10.1016/j.pnpbp.2011.06.013

33. Kim B, Kim CY, Hong JP, Kim SY, Lee C, Joo YH. Brain-derived neurotrophic factor Val/Met polymorphism and bipolar disorder. Association of the Met allele with suicidal behavior of bipolar patients. Neuropsychobiology. 2008;58(2):97-103. doi:10.1159/ 000162356

34. Kim JM, Kang HJ, Bae KY, et al. Association of BDNF promoter methylation and genotype with suicidal ideation in elderly Koreans. Am J Geriatr Psychiatry. 2014;22(10):989-996. doi:10.1016/j. jagp.2014.02.011

35. Van Gastel A, Schotte C, Maes M. The prediction of suicidal intent in depressed patients. Acta Psychiatr Scand. 1997;96(4):254-259.

36. You J, Yuan Y, Shi Y, Zhang Z, Zhang X, Li H. Lack of association between BDNF Val66Met gene polymorphism and late-onset depression in a Chinese Han population. Acta Neuropsychiatr. 2009;21 (4):186-190. doi:10.1111/j.1601-5215.2009.00401.X

37. You J, Yuan Y, Zhang Z, Zhang X, Li H, Qian Y. A preliminary association study between brain-derived neurotrophic factor (BDNF) haplotype and late-onset depression in mainland Chinese. J Affect Disord. 2010;120(1-3):165-169. doi:10.1016/j.jad.2009.04.032

38. Bus BA, Molendijk ML, Penninx BW, et al. Low serum BDNF levels in depressed patients cannot be attributed to individual depressive symptoms or symptom cluster. World J Biol Psychiatry. 2014;15 (7):561-569. doi:10.3109/15622975.2013.841994

39. Dell'Osso L, Del Debbio A, Veltri A, et al. Associations between brain-derived neurotrophic factor plasma levels and severity of the illness, recurrence and symptoms in depressed patients. Neuropsychobiology. 2010;62(4):207-212. doi:10.1159/000319946

40. Lee BH, Kim H, Park SH, Kim YK. Decreased plasma BDNF level in depressive patients. J Affect Disord. 2007;101(1-3):239-244. doi:10.1016/j.jad.2006.11.005

41. Pillai A, Bruno D, Sarreal AS, et al. Plasma BDNF levels vary in relation to body weight in females. PLoS One. 2012;7(7):e39358. doi:10.1371/journal.pone.0039358

42. Piccinni A, Marazziti D, Catena M, et al. Plasma and serum brainderived neurotrophic factor (BDNF) in depressed patients during 1 year of antidepressant treatments. J Affect Disord. 2008;105(13):279-283. doi:10.1016/j.jad.2007.05.005

43. Kreinin A, Lisson S, Nesher E, et al. Blood BDNF level is gender specific in severe depression. PLoS One. 2015;10(5):e0127643. doi:10.1371/journal.pone.0127643

44. Wang XC, Xu DJ, Chen GH, Xia Q, Liu LN. Association of 2 neurotrophic factor polymorphisms with efficacy of paroxetine in patients with major depressive disorder in a Chinese population. Ther Drug Monit. 2014;36(5):612-617. doi:10.1097/FTD.0000000000000062

45. Chi MH, Chang HH, Lee SY, et al. Brain derived neurotrophic factor gene polymorphism (Val66Met) and short-term antidepressant response in major depressive disorder. J Affect Disord. 2010;126 (3):430-435. doi:10.1016/j.jad.2010.07.006 


\section{Publish your work in this journal}

Pharmacogenomics and Personalized Medicine is an international, peer-reviewed, open access journal characterizing the influence of genotype on pharmacology leading to the development of personalized treatment programs and individualized drug selection for improved safety, efficacy and sustainability. This journal is indexed on the American Chemical Society's Chemical Abstracts Service (CAS). The manuscript management system is completely online and includes a very quick and fair peer-review system, which is al easy to use. Visit http://www.dovepress.com/testimonials.php to read real quotes from published authors. 